\title{
CHAPTER «PHYSICAL
}

\section{AND MATHEMATICAL SCIENCES»}

\author{
HIGH ENERGY PHYSICS IN THE CONTEXT OF D-BRANE \\ THEORY AND SEARCHES FOR NEW PHYSICS AT THE LHC
}

\section{Tetiana Obikhod ${ }^{1}$}

DOI: https://doi.org/10.30525/978-9934-588-53-2-58

Abstract. In the last decades, we have witnessed the development of soliton theory. This theory had a significant impact on both physics and mathematics. It provided rich material for models of classical field theory. Among these models there are well-known and important applications of equation such as the Korteweg-de Vries equation, the sine-Gordon equation, and others have proven to be well known. The soliton theory is the fusion of the classical theory of linear differential operators, the theory of Lie algebras, elements of geometry, algebra and the theory of derived categories. The theory of derived categories was the starting point for the development of F-theory, superstring theory, and D-brane theory, a modern version of The Theory of Everything. The paper is devoted to the theoretical researches for high-energy soliton objects of type of D-branes. D-brane theory is characterized by a very rich mathematical apparatus, the components of which are category theory, K-theory and homological algebra, which give information about the spectra, masses, decays of D-branes. For the further studying of D-branes their energetic spectra and phase transitions between different soliton states are calculated. By means of package INSTANTON BPS, states are calculated. For investigation of space of modules of Calabi-Yau manifold - the base of D-brane fiber bundle and for calculation the number of generations of quarks and leptons, the Nakamura's algorithm is applied for calculation the Hilbert scheme for orbifold model. By means of Clifford's algebra, the topological invariant of D-branes - K-functor is calculated, and the decay of D3-brane into

\footnotetext{
${ }^{1}$ Candidate of Physical and Mathematical Sciences, Senior Scientific Researcher, Institute for Nuclear Research of NAS of Ukraine, Kyiv

(C) Tetiana Obikhod
} 
D5-brane and D5-antibrane is studied. The theory of derived categories is applied for investigation of D-brane stability. One of the axioms of the theory of derived categories - the axiom of an octahedron is applied to D-brane treatment of pentaquarks and for investigation the decay of a proton. Within the theory of extra dimensions, microscopic black holes in the deconfinement phase in the framework of AdS/CFT correspondence are considered and found matter content. Experimental observation of high-energy solitonic objects of the D-brane type requires not only a sophisticated and modern mathematical apparatus, but also super expensive and highly efficient experimental complexes. There are many such accelerators, but the Large Hadron Collider is the most promising and effective. The discovery of the Higgs boson is the greatest triumph of experiments at the LHC. However, supersymmetry has been proposed for the solutions of the hierarchy problem. Superstring theory is applied to construct the Minimal Supersymmetric Standard Model. The mass spectrum, partial widths and production cross sections of superpartners are calculated. This approach gives concise predictions for superpartner searches at the LHC. The study of extended Higgs boson sector is made and within Two-Higgs-doublet model the masses, decay widths and production cross sections are calculated. The reconstruction of the LHC at larger energies will make it possible to verify existing predictions of the observables and adjust theoretical models.

\section{Introduction}

Novelty and actuality of theme. The development of high energy physics is now associated with both a powerful theoretical apparatus - the theory of D-branes and superstring, and the ability to test the most recent theoretical predictions in the experiment. Although virtually all of the experimental data observed on the high-energy accelerators are in good agreement with the predictions of the Standard Model (SM), many factors indicate the closeness of the energy scale, at which new physics beyond the SM should manifest. The searches for Kaluza-Klein (KK) particles of gauge bosons, microscopic black holes (MBHs), superpartners, and other exotics in experiments at the Large Hadron Collider (LHC) are aimed at discovering new physics beyond the SM. The increasing accuracy of experimental measurements of observed quantities in modern high energy 
physics requires the improvement of the theoretical description of relevant physical phenomena, which is the main purpose of the work.

The work is devoted to the development and application of D-brane and superstring theory methods to describe BPS states, spaces of extra dimensions, KK partners of gravitons and bosons, superparticles, and the extended Higgs boson sector, the searches for which are realized in modern high energy physics experiments.

The only theory of fundamental interactions is the F-theory, which establishes a one-to-one correspondence between the singularities of the toric varieties and the matter content, which is searched for at the LHC. Typical examples of toric varieties are the Calabi-Yau threefolds, for which the gauge and multiplet content are calculated. The phase transitions between different threefolds give information about the decay and fusion reactions of the particles, which are carried out at high energies. This is very relevant in the light of the search for new physics at the LHC. The use of a mathematical apparatus - homotopy groups, cohomology groups, K-theory for calculating topological invariants of high-energy solitonic objects D-brane, is analogous to the solution of quantum-mechanical equations for finding observable and states. These calculations are a mathematical envelope of those physical phenomena at high energies, the mechanism of which has not yet been established, and where the application of analytical methods to calculate the observed phenomena is an impossible task because of the deep nonlinearity of the processes. Therefore, within the framework of algebraic geometry and theory of derived category, it is important to be able to give preliminary theoretical and computer calculations of topological invariants to characterize solitonic states and to study their transitions to other states, to study the multiplet content of particles, and to understand the strategy of further searches for new physics.

The paper uses the theory of derived category to calculate the physical characteristics of the elementary particles observed in the experiment. Theory of derived category is a mathematical apparatus of superstring theory. Aspinwall's work on the application of category theory to D-branes is a fundamental work in the further application of the mathematical apparatus of derived category theory to the study of stability of D-branes [1]. In high energy physics, we work with ten dimensional space-time $R^{3,1} \times X$, where $R^{3,1}$ is a four-dimensional space-time and $X$ is a space of extra dimensions, 
which, in particular, can be a Calabi-Yau space, an orbifold, etc. We focus our attention on this space and study it in various aspects.

Carrying out mass calculations for KK partners of gravitons and vector bosons within the model of extra dimensions is an urgent task due to the active search for heavy gauge bosons at the CMS and ATLAS (LHC) installations. Experimental search for supersymmetry (SUSY) signal at the LHC within the framework of CMS collaboration at energy $\sqrt{s}_{\mathrm{s}}=13 \mathrm{TeV}$ in proton-proton collisions is important both for the development of methods of experimental search for supersymmetry on CMS and for further search of superparticles at energy $\sqrt{\mathrm{s}}_{\mathrm{s}}=14 \mathrm{TeV}$.

The main purpose of the paper is to study the properties of high-energy solitonic objects such as D-brane and superstrings. The subject of the study is the oscillatory modes of superstrings, i.e., superparticles, the extended Higgs boson sector, the KK partners of gravitons and gauge bosons, microscopic black holes, the purposeful search of which is performed at the LHC. Since the properties of such objects depend on the properties of the space of extra dimensions, the task of the paper is to study the features of such space.

\section{Research methods}

The following methods are used in the paper: toric geometry; algebraic geometry; Nakamura algorithm; K-theory; derived category theory; computer programs INSTANTON, SOFTSUSY, SDECAY, PYTHIA, PROSPINO, HIGLU, SusHi.

String theory appeared as an attempt to describe the strong nuclear force in 1960. The idea of strings was proposed to explain the structure and scattering of $\pi$-mesons at high energies, which were represented as onedimensional strings. Later in 1970, equations of this theory with interactions through strings were proposed by Nambu, Nielsen, Susskind. In 1974, in the framework of string theory the unification of all four fundamental forces and elementary particles was proposed. The incorporation of a supersymmetric group into Neveu-Ramond-Schwarz string model leads Wess and Zumino to the idea of space-time supersymmetry in 1974, the consequence of which was 'Superstring Revolution'. There are five superstring theories: type I with $S O(32)$ gauge symmetry, type IIA and IIB with closed strings and no gauge symmetry, heterotic theory with $S O(32)$ gauge symmetry 
and heterotic theory with $E_{8} \times E_{8}$ gauge symmetry. 'Second Superstring Revolution' in 1995 was proposed by Witten introduced dualities, which unified all five 10-dimensional superstring theories into 11-dimensional M-theory. M-theory led to the concept of D-branes.

The ideology of D-brane theory is connected with the development of the theory of solitons from the equations of Korteweg-de Vries, sine-Gordon to monopoles and instantons. The new solitonic objects that appeared in the 1970 s are the result of superstring theory. Such an interpretation of elementary particles avoided the problems of renormalization of quantum field theory. Incorporation into the theory of open strings led to the introduction of multidimensional objects - Dp-branes ( $p$ - dimension of measurements), on which open strings with Dirichlet boundary conditions are attached. They are solitonic objects in multidimensional space-time and have great application in high energy physics. For D-brane as solitonic object $(X)$, the space-time variety $\mathrm{M}$ is endowed with a certain structure. For the main bundle over $M$

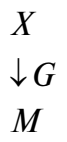

with structural group $G$ as a gauge group, it is possible to construct a vector bundle, which plays an important role not only for the calculations of topological invariants, but also for the study of transitions between D-branes. The basis of the bundle or the space of extra dimensions $M$ may be the space Calabi-Yau, orbifolds and other varieties. The paper is devoted to the study of the properties of Calabi-Yau manifolds, orbifolds, which give information about the physical properties of solitonic states of type of D-brane. For example, Bogomolny-Prasad-Sommerfeld (BPS) states were calculated by Klem, Mair, and Waffa [2] only for threefolds, but fourfolds, which are important in F-theory, were not considered. These manifolds were presented in our paper. The calculation of topological invariants of D-brane with the help of K-theory [3] was proposed by Witten, Olsen, and Szabo [4]. So, the spectra of type IIB and IIA superstrings as the products of the decay of a microscopic black hole calculated using the $\tilde{K}\left(S^{9-p}\right)$ functor and Clifford algebras was actual and considered in the paper. These calculations are important in the aspect of finding of microscopic black hole at high energies at the LHC. 
In 2000, Douglas [5] and Aspinwall [6] constructed the theory of D-branes in terms of derived categories. It is widely used not only for theoretical constructs, but also to calculate the observables. For example, the theory of triangulated categories is applied for the interpretation of the masses of pentaquarks, for the elucidation of proton stability, and to study the stability of solitonic objects at high energies.

The F-theory, which combines four types of interactions at high energies is now being tested at the LHC. Particles such as microscopic black holes, KK partners of gravitons and gauge bosons are the subject of search at the ATLAS and CMS experimental facilities at the LHC. Within the framework of AdS/CFT correspondence, the paper presents the searches for a microscopic black hole in deconfinement phase. The matter content described by the Conformal Field Theory (CFT) which is holographically dual to the space of anti-de-Sitter (AdS) is obtained.

The transition of the F-theory to the supersymmetric theory at energies of $10^{15} \mathrm{GeV}$ allows us to consider the MSSM model. The search for superparticles, as a confirmation of the theory of SUSY, is being actively pursued at the LHC.

The discovery of the Higgs boson in 2012 [7; 8] was the most important event in physics in recent years. Now, at the LHC the properties of this particle are studied. Although it is confirmed that it belongs to the SM, but some of its properties are uncertain. MSSM theory predicts the existence of an extended Higgs boson sector. Within the framework of this theory, we calculated the properties of the SM-like Higgs boson.

\section{F-theory and properties of Calabi-Yau manifolds and orbifolds}

F-theory as theory of all particles and interactions generalizes superstring theory and called The Theory of Everything (TOE). Modern physics requires that the TOE combine four known fundamental interactions: gravitational interaction, electromagnetic interaction, strong nuclear force, weak nuclear force.

The first step towards this was to combine electromagnetic and weak interactions in the theory of electroweak interaction, created in 1967 by Stephen Weinberg, Sheldon Glashow, and Abdus Salam. In 1973, the theory of strong interaction was proposed. Currently, the main candidate for TOE is the F-theory, which operates with more than the observed 3+1 space-time 
dimensions. The impetus for this has been the Kaluza-Klein theory, which allows the integration of general theory of relativity and electromagnetic theory by introducing an extra dimension.

The F-theory is a twelve-dimensional theory that is defined at energies of the order of $10^{19} \mathrm{GeV}$. The compactification of the F-theory to the space of extra dimensions leads to a our four-dimensional space with certain characteristics of solitonic states in vacuum. Therefore, to study the supersymmetry defined in four-dimensional space, we consider the compactification of the F-theory on Calabi-Yau manifolds with certain properties. The large number of Calabi-Yau manifolds leads to a large number of new models being implemented in the low-energy approximation. Therefore, one of the main tasks of the physicists is to select from a satisfactory set of spaces Calabi - Yau such set that would give the most adequate justification of the number and content of all known particles. The physical characteristics of topological solitonic states, which are realized as elementary particles in a low-energy approximation, are determined by studying the features of the Calabi-Yau manifolds using algebraic geometry [9].

Our aim is to calculate the spectra of BPS states in F-theory on elliptically fibered fourfolds using the computer program INSTANTON.

Let us consider the Donagi-Grassi-Witten Model. The spectrum of BPS states is presented in Table 1 for the model $\mathrm{P}^{1} \times S$, where $\mathrm{P}^{1}-$ the onedimensional projective space, $S$ - the del Pezzo surface.

The sequences, $1 \cdot 252 *, 2 \cdot 5130 *, 3 \cdot 54760 *$, (marked with an asterisk in Table 1) are new and physically interesting because they indicate the presence of additional states.

We also considered elliptically fibered fourfold over $\mathrm{P}^{1}$-fibration $\mathrm{P}\left(\mathrm{O}_{B} \otimes \mathrm{O}_{B}\left(f_{1}+f_{2}\right)\right)$ on the base $B=\left(\mathrm{P}^{1}\right)^{2}$, where $f_{1}$ and $f_{2}$ are the layers of two projections from $B$ in $\mathrm{P}^{1}$ and elliptically fibered fourfold over $\mathrm{P}^{1} \times F_{1}$, where $F_{1}$ is the Hirzebruch surface. In these two cases sequences

$$
\begin{gathered}
1 \cdot 252,2 \cdot 5130,3 \cdot 54760, \ldots, \\
-2 \cdot 9252,-3 \cdot 673760,-4 \cdot 20534040, \ldots, \\
3 \cdot 848628,4 \cdot 115243155,5 \cdot 6499779552, \ldots
\end{gathered}
$$

are new and interesting from a physical point of view as they indicate the presence of additional states. 
Table 1 Instantaneous numbers (degeneracy rates of BPS states) for the model $P^{1} \times S$

\begin{tabular}{|c|c|c|c|c|c|}
\hline $\mathrm{n}_{2,1,3,0,0}$ & $\mathrm{n}_{2,1,3,0,1}$ & $\mathrm{n}_{2,1,3,0,2}$ & $\mathrm{n}_{2,1,3,0,3}$ & $\mathrm{n}_{2,1,3,0,4}$ & $\mathrm{n}_{2,1,3,0,5}$ \\
\hline 1 & 252 & 5130 & 54760 & 419895 & 2587788 \\
\hline \multicolumn{6}{|c|}{27 sequences } \\
\hline $\mathrm{n}_{2,1,3,0,1}$ & & $\mathrm{n}_{2,2,3,0,1}$ & $\mathrm{n}_{2,3,3,0,1}$ & $\mathrm{n}_{2,4,3,0,1}$ & $\mathrm{n}_{2,5,3,0,1}$ \\
\hline $1 \cdot 252 *$ & & $2 \cdot 5130 *$ & $3 \cdot 54760 *$ & $4 \cdot 419895 *$ & $5 \cdot 2587788 *$ \\
\hline \multicolumn{6}{|c|}{27 sequences } \\
\hline $\mathrm{n}_{4,0,3,0,1}$ & $\mathrm{n}_{4,1,3,0,1}$ & $\mathrm{n}_{4,2,3,0,1}$ & $\mathrm{n}_{4,3,3,0,1}$ & $\mathrm{n}_{4,4,3,0,1}$ & $\mathrm{n}_{4,5,3,0,1}$ \\
\hline 1 & 252 & 5130 & 54760 & 419895 & 2587788 \\
\hline \multicolumn{6}{|c|}{513 sequences } \\
\hline $\mathrm{n}_{5,0,3,0,1}$ & $\mathrm{n}_{5,1,3,0,1}$ & $\mathrm{n}_{5,2,3,0,1}$ & $\mathrm{n}_{5,3,3,0,1}$ & $\mathrm{n}_{5,4,3,0,1}$ & $\mathrm{n}_{5,5,3,0,1}$ \\
\hline 1 & 252 & 5130 & 54760 & 419895 & 2587788 \\
\hline \multicolumn{6}{|c|}{702 sequences } \\
\hline $\mathrm{n}_{6,0,3,0,1}$ & $\mathrm{n}_{6,1,3,0,1}$ & $\mathrm{n}_{6,2,3,0,1}$ & $\mathrm{n}_{6,3,3,0,1}$ & $\mathrm{n}_{6,4,3,0,1}$ & $\mathrm{n}_{6,5,3,0,1}$ \\
\hline 1 & 252 & 5130 & 54760 & 419895 & 2587788 \\
\hline \multicolumn{6}{|c|}{189 sequences } \\
\hline
\end{tabular}

The study of D-branes on orbifolds is connected with the space of the orbifold modules $C^{3} / Z_{n}, n=3$. It is constructed using the Nakamura algorithm. An example is Nakamura's algorithm for a model $\frac{1}{3}(1,1,1)$ is presented by the McKay quiver parked with tripods in Figure 1 and Figure 2.

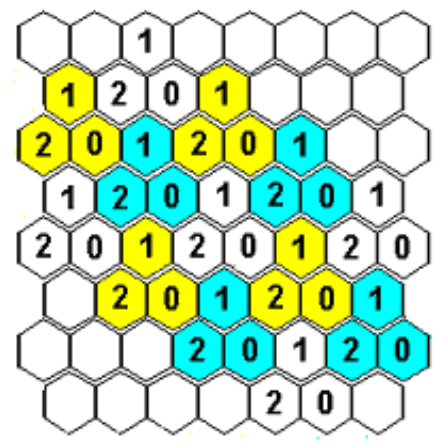

Figure 1. McKay quiver for the model $\frac{1}{3}(1,1,1)$

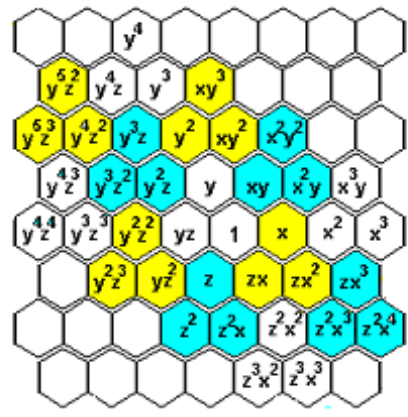

Figure 2. A monomial representation of the McKay quiver for the model $\frac{1}{3}(1,1,1)$ 


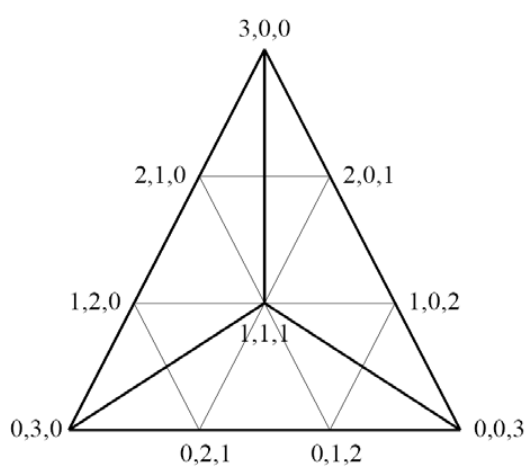

Figure 3. The Hilbert scheme of the model $\frac{1}{3}(1,1,1)$

Hilbert's scheme for the model $\frac{1}{3}(1,1,1)$ is presented in Figure 3 .

Thus, the presentation of the Hilbert scheme as a toric variety of fans and the splitting of the fans into cones has a deep physical meaning. For example, in the case of a model $\frac{1}{3}(1,1,1)$, knowing the number of vertices of the Hilbert scheme and the number of cones, we have information on the fundamental representation of the group SU(3). The number of generations of quarks and leptons characterized by this group is equal to three.

\section{D-brane properties and high energy physics}

The section is devoted to the study of D-brane properties. D-branes as solitonic objects are characterized by topological invariants of the type of homotopy groups or cohomology groups [10]. At high energies, instead of the analytical approach, it is advisable to switch to the fibered spaces for calculations of topological invariants, which are analogues of the observables in quantum physics. We deal with the computation of

\section{Cliffford algebras and groups $\bar{K}\left(S^{k}\right)$}

\begin{tabular}{|c|c|c|}
\hline & $C_{k}^{c}$ & $\bar{K}\left(S^{k}\right)$ \\
\hline 1 & $C \oplus C$ & 0 \\
\hline 2 & $C(2)$ & $Z$ \\
\hline 3 & $C(2) \oplus C(2)$ & 0 \\
\hline 4 & $C(4)$ & $Z$ \\
\hline 5 & $C(4) \oplus C(4)$ & 0 \\
\hline 6 & $C(8)$ & $Z$ \\
\hline 7 & $C(8) \oplus C(8)$ & 0 \\
\hline 8 & $C(16)$ & $Z$ \\
\hline
\end{tabular}


topological charges of D-branes using the K-theory. To consider the decay of the D-branes the Clifford algebra together with the Grotendick group $K$ is applied. This group describes the topological charges of a topological soliton of D-brane type presented in Table 2.

The spectrum has been calculated by applying the spectral sequence of Ati-Hirzebruch. The decay of the D3-brane corresponds to the nesting of K-groups

$$
\bar{K}\left(S^{6}\right) \supset \bar{K}\left(S^{4}\right) \oplus \bar{K}\left(S^{4}\right)
$$

which signals about the existence of a decay

$$
D 3 \rightarrow D 5+\overline{D 5} \text {. }
$$

The consideration of the triangulated category and octahedron diagram
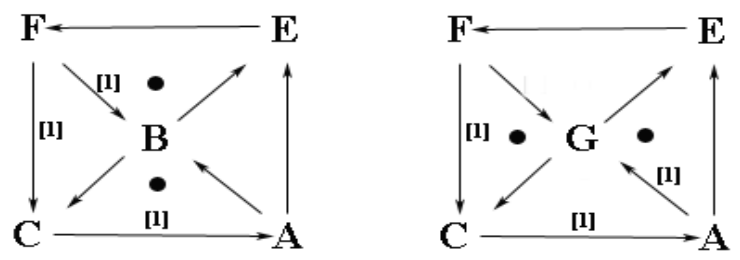

where distinguished triangles are marked by $\bullet$.

Since quarks, leptons, and $\mathrm{X}$-bosons are solitonic excitations in a proton, we plot an octahedron
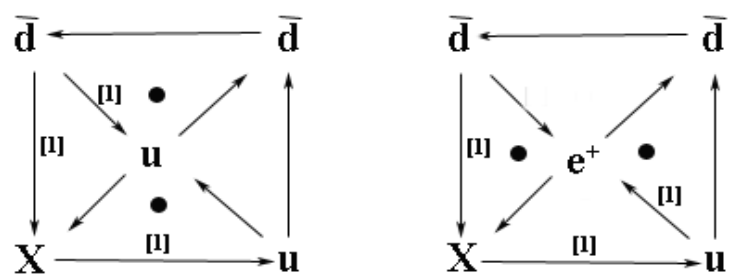

From the consideration of the experimental data on masses of u-quark and d-quark [11] we conclude that the proton decay is impossible.

\section{The study of the properties of Kaluza-Klein modes}

Another solutions to the problem of the hierarchy are models with extra dimensions: Arkani-Hamed-Dimopoulos-Dvali (ADD) [12] and Randall- 
Sundrum (RS) [13]. One of the characteristic features of the models with extra dimensions is the existence of the Kaluza-Klein (KK) mode. Their identification is one of the leading tasks of the LHC. It is assumed that during the collision of $14 \mathrm{TeV}$ particles, a short-lived fireball or a microscopic black hole $(\mathrm{mbh})$ is formed in the center of mass energy system, which decays into a jets of new particles $-\mathrm{KK}$ - partners.

We have considered the microscopic black hole in the deconfinement phase in the framework of AdS/CFT correspondence. The matter content, described by holographically dual to the AdS space Young-Mills theory, has the following form

$$
\begin{aligned}
& D(1,0,0 \mid 6)_{0}+D\left(\frac{3}{2}, \frac{1}{2}, 0 \mid 4\right)_{2}+D\left(\frac{3}{2}, 0, \frac{1}{2} \mid \overline{4}\right)_{2}+D(2,1,0 \mid 1)_{2}+D(2,0,1 \mid 1)_{2}, \\
& D(4,1,1 \mid 1)_{0}+D\left(\frac{7}{2}, 1, \frac{1}{2} \mid \overline{4}\right)_{2}+D\left(\frac{7}{2}, \frac{1}{2}, 1 \mid 4\right)_{2}+D\left(3, \frac{1}{2}, \frac{1}{2} \mid 15\right)_{1}+D(3,1,0 \mid 6)_{2}+ \\
& +D(3,0,1 \mid \overline{6})_{2}+D\left(\frac{7}{2}, \frac{1}{2}, 0 \mid 4\right)_{2}+D\left(\frac{7}{2}, 0, \frac{1}{2} \mid \overline{4}\right)_{2}+D\left(\frac{5}{2}, \frac{1}{2}, 0 \mid 20\right)_{2}+D\left(\frac{5}{2}, 0, \frac{1}{2} \mid \overline{20}\right)_{2}+ \\
& +D(2,0,0 \mid 20)_{0}+D(3,0,0 \mid 10)_{0}+D(3,0,0 \mid \overline{10})_{0}+D(4,0,0 \mid 1)_{0}+D(4,0,0 \mid 1)_{0} .
\end{aligned}
$$

and is described by both exotic KK partners and well-known 15-plet of vector mesons $D\left(3, \frac{1}{2}, \frac{1}{2} \mid 15\right)_{1}$ and 20 -plet of baryons $D\left(\frac{5}{2}, \frac{1}{2}, 0 \mid 20\right)_{2}$, which emphasizes the correct choice of theory to predict the spectrum of particles, purposeful searches for which are carried out at the LHC.

\section{Searches for superparticles}

The search for new physics at the LHC is intended to discover superpartners of quarks and leptons provided by MSSM model. As SUSY model is the part of unified theory of fundamental interactions, we must build MSSM model by using theories of D-branes and superstrings. The theory of D-branes and superstrings is based on the mathematical apparatus of the theory of derived categories. In this approach, D-branes are described by quivers, and superstrings are described by Ext groups. The objects of derived category are quivers, and morphisms are the Ext-groups that describe the superstrings. Ext-group calculations make it possible to calculate the particle content of the MSSM model, which, in addition to the known 
particles, contains superpartners and extended sector of Higgs bosons. Computer algorithms allows us to calculate the mass spectrum of superpartners and Higgs bosons, as well as the partial widths and production cross sections of the superparticles. These predictions are very promising because they are related to the search for new physics beyond the SM and can be tested on the CMS and ATLAS detectors. The quivers and morphisms are presented in Figure 4.

Substitution of orbifold charges

$$
a=b=c=a^{\prime}=b^{\prime}=c^{\prime}=4
$$

in groups

$$
\begin{aligned}
& \operatorname{Ext}^{0}\left(Q, Q^{\prime}\right)=C^{a a^{\prime}+b b^{\prime}+c c^{\prime}}, \\
& \operatorname{Ext}^{1}\left(Q, Q^{\prime}\right)=C^{3 a b^{\prime}+3 b c^{\prime}+3 c a^{\prime}}
\end{aligned}
$$
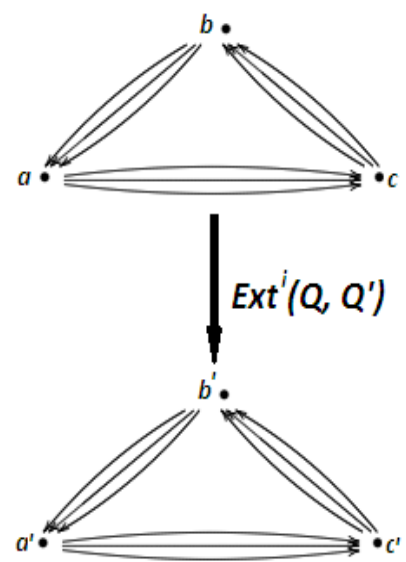

Figure 4.

The representation of

D-branes by quivers and superstrings by arrows

together with the use of the Langlands hypothesis [14], gives the realization of the space of superstring modules in terms of $\mathrm{SU}(5)$ multiplets:

$$
3 \times\left(24+5_{H}+\overline{5}_{H}+5_{M}+\overline{5}_{M}+10_{M}+\overline{10}_{M}\right) .
$$

With this result, we can construct a gauge invariant superpotential of the MSSM model

$$
W_{S U(5)}=\lambda_{i j}^{d} \cdot \overline{5}_{H} \times \overline{5}_{M}^{(i)} \times 10_{M}^{(j)}+\lambda_{i j}^{u} \cdot 5_{H} \times 10_{M}^{(i)} \times 10_{M}^{(j)}+\mu \cdot 5_{H} \times \overline{5}_{H} .
$$

The use of this superpotential, together with the five parameters on which it depends, makes it possible to calculate the masses of superparticles, the widths of decays, the production cross sections of the superparticles, the histograms of the mass distribution of superparticles. An example of calculating the partial widths of superpartners for gluino is shown in Table 3.

The histogram of the mass distribution of superparticles, calculated with the corresponding five parameters

$$
\begin{aligned}
& m_{0}=20 \mathrm{GeV}, m_{1 / 2}=440 \mathrm{GeV}, A_{0}=-25 \mathrm{GeV}, \\
& \tan \beta=15, \operatorname{sgn}(\mu)=+1
\end{aligned}
$$

is presented in Figure 5. 
Table 3

Partial widths of superpartners for gluino

\begin{tabular}{|c|lc|ll|}
\hline & channel & BR & channel & BR \\
\hline$\tilde{g}$ & $\tilde{d}_{L} d^{*}$ & 0.023 & $\tilde{c}_{L} c^{*}$ & 0.025 \\
& $\tilde{d}_{L}^{*} d$ & 0.023 & $\tilde{c}_{L}^{*} c$ & 0.025 \\
& $\tilde{d}_{R} d^{*}$ & 0.046 & $\tilde{c}_{R} c^{*}$ & 0.044 \\
& $\tilde{d}_{R}^{*} d$ & 0.046 & $\tilde{c}_{R}^{*} c$ & 0.044 \\
& $\tilde{u}_{L} u^{*}$ & 0.025 & $\tilde{b}_{1} b^{*}$ & 0.074 \\
& $\tilde{u}_{L}^{*} u$ & 0.025 & $\tilde{b}_{1}^{*} b$ & 0.074 \\
& $\tilde{u}_{R}^{*} u^{*}$ & 0.044 & $\tilde{b}_{2} b^{*}$ & 0.050 \\
& $\tilde{u}_{R}^{*} u$ & 0.044 & $\tilde{b}_{2}^{*} b$ & 0.050 \\
& $\tilde{u}_{R}^{*} b$ & 0.099 \\
& $\tilde{s}_{L} s^{*}$ & 0.023 & $\tilde{t}_{1} t^{*}$ & 0.099 \\
& $\tilde{s}_{L}^{*} s$ & 0.023 & $\tilde{t}_{1}^{*} t$ & \\
& $\tilde{s}_{R} s^{*}$ & 0.046 & & \\
& $\tilde{s}_{R}^{*} s$ & 0.046 & & \\
\hline
\end{tabular}

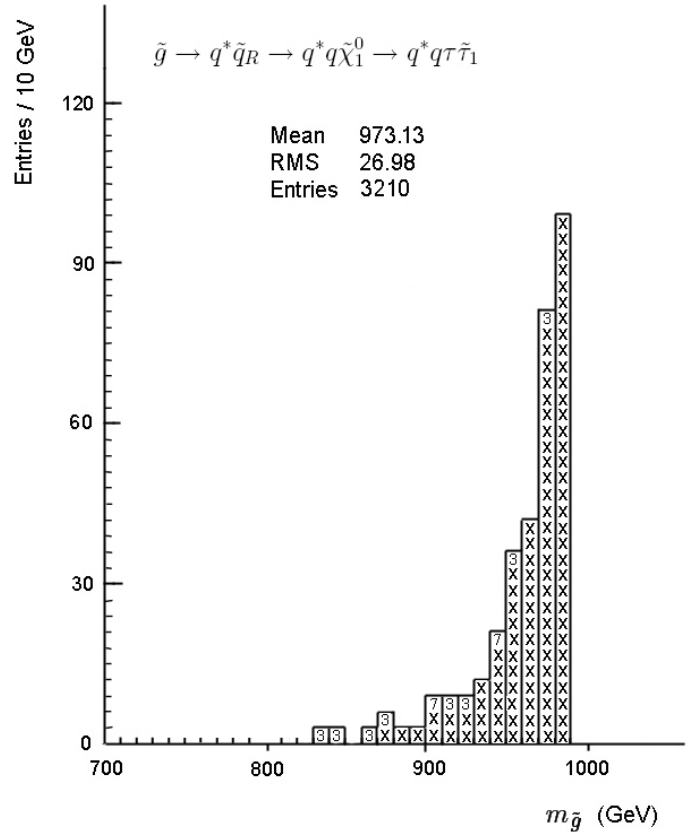

Figure 5. The histogram of the mass distribution of gluino $\tilde{g}$ 


\section{The study of the Higgs boson properties within MSSM model}

After the discovery of the Higgs boson in 2012, in the physics of elementary particles began a comprehensive study of its properties. The main goal is to study the properties of this particle, its deviation from the predictions of SM. The detection of physics beyond the SM connected with many scenarios which include a SM-like Higgs boson as part of an extended sector of scalar particles. In any case, searches for new Higgs bosons are connected with the measurements of the properties of the new particles of an extended sector.

With the help of SOFTSUSY, SDECAY, HIGLU computer programs, within the experimentally selected set of MSSM model parameters, masses, decay widths and production cross sections of the lightest Higgs boson of MCCM model were calculated. It is shown that the mass at a certain set of parameters coincides with the mass of the experimentally open Higgs boson (Table 4).

Table 4

The mass spectrum of two Higgs bosons $M_{h}, M_{A}$ of MSSM model

\begin{tabular}{|c|c|c|c|c|c|}
\hline $\boldsymbol{m}_{\mathbf{0}}$ & $\boldsymbol{m}_{\mathbf{1 / 2}}$ & $\tan \boldsymbol{\beta}$ & $\boldsymbol{A}_{\mathbf{0}}$ & $\boldsymbol{M}_{\boldsymbol{h}}$ & $\boldsymbol{M}_{\boldsymbol{A}}$ \\
\hline 800 & 650 & 10 & 0 & 116 & 118 \\
\hline 800 & 650 & 50 & 0 & 117 & 620 \\
\hline 800 & 650 & 50 & 50 & 117 & 618 \\
\hline 800 & 650 & 50 & 300 & 117 & 609 \\
\hline 800 & 1500 & 10 & 0 & 120.9 & 205.5 \\
\hline 800 & 2000 & 10 & 0 & 122.2 & 258.8 \\
\hline 800 & 2500 & 10 & 0 & 123.1 & 311.8 \\
\hline 2000 & 2500 & 10 & 0 & 123.2 & 356.5 \\
\hline 2000 & 3000 & 50 & 0 & 124.2 & 212.1 \\
\hline 3000 & 3000 & 30 & 0 & 124.7 & 385.7 \\
\hline 3000 & 3000 & 25 & 0 & 124.8 & 407.7 \\
\hline
\end{tabular}

One of the models of beyond the SM physics is the two-Higgs doublet (2HDM) model [15]. This model provides a solution to the hierarchy problem and predicts five Higgs bosons: two neutral CP-even Higgs bosons, $\mathrm{h}$ and $\mathrm{H}$, one neutral CP-odd Higgs, A and two charged Higgs bosons, $H^{ \pm}$. There are many experimental data connected with searches for extended sector 
of Higgs bosons, one of which is the resonant Higgs boson production via the process $p p \rightarrow H \rightarrow h h \rightarrow b b \tau \tau$, where $\mathrm{H}$ is the CP-even Higgs boson of unknown mass, Figure 6.

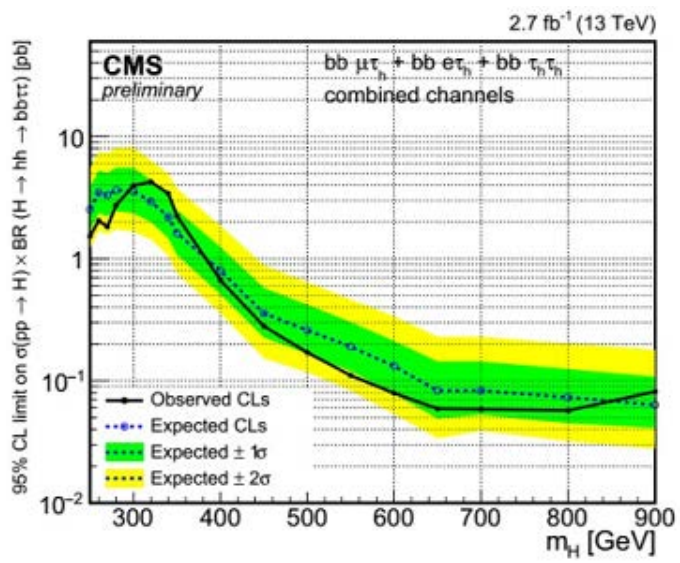

Figure 6. The upper limit on $\sigma(p p \rightarrow H) \times B R(H \rightarrow h h \rightarrow b b \tau \tau)$ as the function of $m_{H},[16]$.

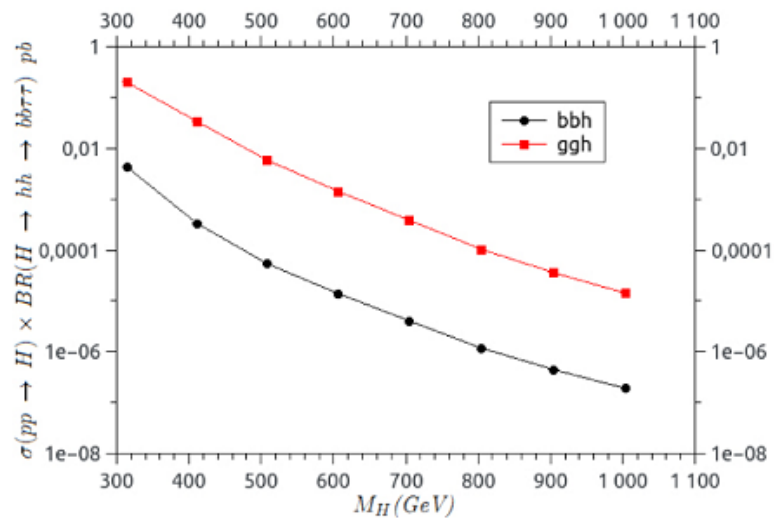

Figure 7. $\sigma(p p \rightarrow H) \times B R(H \rightarrow h h \rightarrow b b \tau \tau)$ for the gluon-gluon fusion $(g g \varphi)$ and b-associated production process $(b b \varphi)$ at a center-of-mass energy of $14 \mathrm{TeV}$ at the $\mathrm{LHC}$ 
Using the computer program SusHi, we calculated $\sigma(p p \rightarrow H) \times B R(H \rightarrow h h \rightarrow b b \tau \tau)$ for the gluon-gluon fusion $(g g \varphi)$ and b-associated production process ( $b b \varphi$ ) at a centre-of-mass energy of $14 \mathrm{TeV}$ and $\tan \beta=2$ in the mass range $\mathrm{M}_{\mathrm{H}}=300-1000 \mathrm{GeV}$, Figure 7 .

Production via gluon-gluon fusion and b-associated production process and found the predominance of the ( $g g \varphi$ ) process for small values of $\tan \beta$.

\section{Conclusions}

The paper is devoted to the study of the properties of D-branes and to the searches for new physics beyond the SM at the LHC. Since the properties of the D-brane are determined by the properties of the space of extra dimensions, which carries information about particles that are formed at high energies, the following work has been done to study this space using various mathematical methods:

- the instanton numbers for the three models of Calabi-Yau fourfolds are calculated and found new sequences of BPS states;

- Nakamura's algorithm applied to calculate Gilbert's scheme for orbifold $C^{3} / Z_{n}$, which is associated with the procedure of blowing up of singularity. With module space represented in the form of a Gilbert scheme, a set of particles is associated;

- using the K-theory has made it possible to perform the calculations of Grotendick K-group by means of Clifford algebras do predict the decay of D3-brane into D5- and $\overline{D 5}$-branes;

- in the framework of the theory of derived category using the triangulated categories it has been possible to calculate masses of pentaquarks and to elucidate the proton stability conditions;

- microscopic black holes in the deconfinement phase in the framework of AdS/CFT correspondence are considered. The matter content described by the supersymmetric Young-Mills theory is obtained;

- within the supersymmetric MSSM model using computer programs the following observables the following parameters are calculated: masses, widths of decays, production cross sections, histograms of mass distribution of superparticles;

- within 2HDM model the production of cross sections on branching fraction, $\sigma(p p \rightarrow H) \times B R(H \rightarrow h h \rightarrow b b \tau \tau)$ at $14 \mathrm{TeV}$ with the help of a computer program SusHi have been calculated. 


\section{References:}

1. P.S. Aspinwall. D-branes on Calabi-Yau manifolds // e-print arXiv: hep-th/0403166.

2. A. Klemm, P. Mayr, C.Vafa. BPS states of exceptional non-critical strings. Harvard, 1996. - 29 p. - (Preprint, HUTP-96/A031).

3. M.F. Atiyah, F. Hirzebruch. Vector bundles and homogeneous spaces // Amer. Math. Soc. Proc. Symp. Pure Math. - 1961. - 3. - P. 7-38.

4. K. Olsen, R. J. Szabo. Constructing D-branes from K-theory // Adv. Theor. Math. Phys. - 1999. - 3. - P. 889-1025.

5. M.R. Douglas. D-branes, categories and $\mathrm{N}=1$ supersymmetry // J. Math. Phys. $-2001 .-42$. - P. 2818-2843.

6. P.S. Aspinwall, A.E. Lawrence. Derived categories and zero-brane stability // JHEP. - 2001. - 0108:004. - $26 \mathrm{p}$.

7. ATLAS collaboration. Observation of a new particle in the search for the Standard Model Higgs boson with the ATLAS detector at the LHC // Phys. Lett. B - 2012. - 716. - P. 1-29.

8. CMS collaboration. Observation of a new boson at a mass of $125 \mathrm{GeV}$ with the CMS experiment at the LHC // Phys. Lett. - 2012. - B716. - P. 30-61.

9. Hirzebruch. Topological methods in algebraic geometry. - New York: Springer-Verlag, 1966. - $280 \mathrm{p}$.

10. S.I. Gelfand, Yu.I. Manin. Homological algebra. - Berlin: Springer-Verlag, 1994. $-416 \mathrm{p}$.

11. A.V. Manohar, C.T. Sachrajda. Quark masses // Phys. Rev. - 2002. - D66. P.010001.

12. N. Arkani-Hamed, S. Dimopoulos, G. Dvali. Phenomenology, astrophysics and cosmology of theories with sub-millimeter dimensions and $\mathrm{TeV}$ scale quantum gravity // Phys. Rev. - 1999. - D59, N 8. - P. 086004.

13. L. Randall, R. Sundrum. A Large mass hierarchy from a small extra dimension // Phys. Rev. Lett. - 1999. - 83. - P. 3370-3373.

14. W. Schmid. Homogeneous complex manifolds and representations of semisimple Lie groups // Proc. Natl. Acad. Sci. USA. - 1968. - 59, N1. - P. 56-59.

15. G.C. Branco et al. Theory and phenomenology of two-Higgs-doublet models // Phys. Rept. 2012, 516, p. 1-102.

16. CMS Collaboration. Search for resonant Higgs boson pair production in the bb $\tau \tau$ final state // CMS-PAS-HIG-16-013. 